\title{
Laparoscopic Excision of A Rare Retroperitoneal Non-Pancreatic Pseudocyst
}

\author{
Sardar Rezaul Islam* \\ Shafiqur Rahman \\ Kamal Pasha \\ ASM Sayem
}

Department of Surgery

Jahurul Islam Medical College \&hospital

Kishoregoni, Bangladesh.

Correspondence to:

\section{Dr. Sardar Rezaul Islam}

Associate Professor

Department of Surgery

Jahurul Islam Medical College \& Hospital

Kishoregoni, Bangladesh.

Mobile: +880-1746603305

E-mail: islamreza@hotmail.com

\begin{abstract}
Retroperitoneal cysts (RCs) are rare. They are composed of both epithelial and mesothelial tissues, and those without an epithelial lining in the wall are called pseudocysts. Most retroperitoneal pseudocysts are pancreatic in origin, and nonpancreatic pseudocysts are very rarely reported.We report a case of large cystic lesion adjacent to the left kidney in a 25 years old man. He presented with 2 and half years history of gradual swelling of left upper abdomen. Abdominal ultrasonography and CT scan showed a large unilocular cystic mass in left side of the abdomen, which was attached with the lower pole of the left kidney. The cystic mass was excised laparoscopically. Histology showed a fibrous wall without epithelial lining thus confirming the diagnosis of a nonpancreatic pseudocyst.
\end{abstract}

Key words : Retroperitoneal cysts; non-pancreatic pseudocysts; laparoscopic excision.

\section{INRODUCTION}

Retroperitoneal cystic (RC) masses are relatively rare in clinical settings. There are no specific clinical signs for RCs, although vague abdominal pain and disten tion are present in $50 \%$ of cases with an $\mathrm{RC}^{1}$. They usually attain a large mass before causing any symptoms. Therefore, they are often accidentally discovered ${ }^{2}$. RCs without an epithelial lining in the wall are called pseudocysts. Most of the retroperitoneal pseudocysts are of pancreatic origin.

Cystic lesions of the retroperitoneum are pathologically classified as either neoplastic or non-neoplastic. Neoplastic lesions include cystic lymphangioma, mucinous cystadenoma, cystic teratoma, cystic mesothelioma, müllerian cyst, epidermoid cyst, tail gut cyst, bronchogenic cyst, cystic change in solid neoplasms, pseudomyxoma retroperitonei, and perianal mucinous carcinoma. Non-neoplastic lesions include pancreatic pseudocyst, nonpancreatic pseudocyst, lymphocele, urinoma, and hematoma ${ }^{3-4}$.

\section{CASE HISTORY}

A 25 years old man, who lives abroad, presented with gradual abdominal distension and a swelling over a period of two and a half year. He did not give any history of trauma. He had no alteration of bowel habit. There was neither urinary symptom nor any history of fever. On examination there was smooth non tender swelling felt over the left side of the lumber area. Ultrasonography of the abdomen detected a large cyst in the abdomen in front of the left kidney. The diameter of the cyst was $9.6 \mathrm{~cm} \times 9.0 \mathrm{~cm}$. Subsequent CT scan confirmed a unilocular cyst, which has thick wall and contains dense fluid. The cyst was completely separated from the spleen and pancreas but has deep adherence with the lower pole of the left kidney on CT (Fig-1). IVU did not show any communication with the collecting system of the kidney. The patient was non diabetic. His TLC was $14000 / \mathrm{cu} \mathrm{mm}, \mathrm{Hb} 14.1 \mathrm{gm}$, ESR $15 \mathrm{~mm} / 1 \mathrm{st}$ hour and serum creatinine $1 \mathrm{mg} / \mathrm{dl}$. His chest $\mathrm{x}$-ray was normal. USG guided FNAC showed serous fluid and no evidence of malignancy. We decided to excise the cyst laparoscopically. 
The patient was operated on semi-right lateral position. Three trochars were placed as shown in the picture. Two 10 $\mathrm{mm}$ and one $5 \mathrm{~mm}$ trochars were used. During surgery cyst was punctured and aspirated in order to reduce the size of the cyst. Very thick serous fluid was found. The cyst was successfully separated from the surrounding structure such as spleen, descending colon and anterior wall of the kidney. At the lower pole of the left kidney, for an area of approximately $4 \mathrm{sq} \mathrm{cm}$, the cyst was found to be deeply adherent. At that point the cyst and the capsule of the left kidney were inseparable. So cyst was excised leaving the capsule of the kidney intact. The patient recovered uneventfully except a minor wound infection at one of the trocar site. Patient was discharged on $5^{\text {th }}$ postoperative day. He was seen on follow up. The trocar sites have healed. The patient was found to be very satisfied with the surgery (Fig2). He left for abroad and joined his duty in three weeks time.

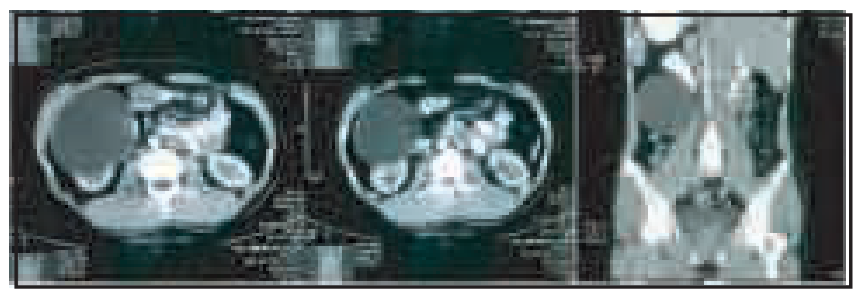

Figure 1: Preoperative CT scan of abdomen

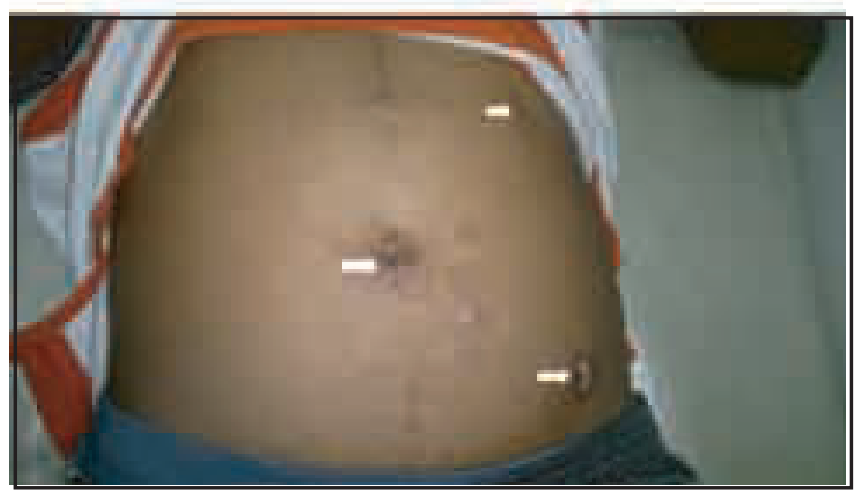

Figure 2: Postoperative photo of port sites

\section{DISCUSSION}

RCs were defined by Handfield-Jones as cysts existing in the retroperitoneal fatty tissues that have no connection with any adult anatomic structure except areolar tissue ${ }^{5}$. Their precise pathogenesis remains unknown, but many possible pathologic processes have been proposed that can roughly be divided into urogenital, mesocolic, teratomatous, parasitic, traumatic, and lymphatic ${ }^{6}$.
A pseudocyst differs from a cyst in the absence of any epithelial lining. According to literature reports, pseudocysts commonly originate from the pancreas and develop from acute pancreatitis ${ }^{7-8}$. Unlike pancreatic pseudocysts, non-pancreatic pseudocysts are not associated with high levels of amylase or lipase in the cystic fluid. They usually has a thick fibrous wall and contain blood, pus, or serous fluid. Because there are no clinically characteristic symptoms or signs for RCs, clinicians should consider the possibility of an RC when they confront a patient who presents with vague abdominal pain and distension and when a palpable abdominal mass is demonstrated. In some circumstances, the patient with an RC may occasionally present with acute abdominal pain when the RC becomes hemorrhagic or infected. CT is ideal for assessing RCs because it provides discrete sectional images of the organs and retroperitoneal compartments. Characteristics of non-pancreatic pseudocysts are clearly manifested on CTscans as unilocular or multilocular fluidfilled masses with thick walls ${ }^{9}$. Histologic features of RCs are important for making final diagnosis. On the microscopic analysis, the walls of pseudocysts consist of dense fibrous tissue (the mesothelium) with no epithelial lining. They are similar to the histologic features of lymphoceles. The differential diagnosis between them is based on the history, imaging and fluid contents. As mentioned above, our patient had no surgical or traumatic history. Lymphoceles were not likely as the cyst did not contain chylous fluid. The treatments of choice for RCs include complete excision and simple drainage. Excision of cysts is the best policy because of their potential recurrence. Possible surgical approach includes open laparotomy or a laparoscopic excision. As the cyst was moderately large and protruded anteriorly, laparoscopic approach seemed feasible. We used semi-right lateral position of the patient after insertion of the first trocar and creation of pneumoperitoneum. This helped left colon and small intestine to fall away from the cyst. Recurrence following excision of a retroperitoneal cyst can occur if the excision is incomplete. In an analysis of the 23 patients who had an RC that the recurrent rate was about $22 \%$ (five recurrences occurred in 23 patients ${ }^{10}$. In our case, excision was complete and there was no recurrence in the short period of follow up. 


\section{CONCLUSION}

Retroperitoneal non-pancreatic pseudocysts are very rare. We presented this case of progressive lower abdominal pain that was ultimately diagnosed as a retroperitoneal nonpancreatic pseudo-cyst. These kind of retroperitoneal or peri-renal cysts can be successfully excised laparoscopically.

\section{DISCLOSURE}

All the authors declared no competing interest.

\section{Reference}

1. Adams JT, Schwartz SI, Shires GT, Spencer FC. Abdominal wall, omentum, mesentery and retroperitoneum. editors. Principles of surgery . 5th ed. NewYork, NY: McGraw-Hill; 1989;1491-524.

2. Pace G, Galatioto Paradiso G, Galassi P, Vicentini C. Retroperitoneal cysts: a case report. Arch Ital Urol Androl 2006; 78: 25-6.

3. Ros PR, Olmsted WW, Moser RP, Dachman AH, Hjermstad BH. Mesenteric and omental cysts: histologic classification with imaging correlation. Radiology 1987; 164: 327-332.

4. Oray-Schrom P, St Martin D, Bartelloni P, AmoatengAdjepong Y. Giant nonpancreatic pseudocyst causing acute anuria. J Clin Gastroenterol 2002; 34: 160-163.
4. Oray-Schrom P, St Martin D, Bartelloni P, AmoatengAdjepong Y. Giant nonpancreatic pseudocyst causing acute anuria. J Clin Gastroenterol 2002; 34: 160-163.

5. Handfield-Jones RM. Retroperitoneal cysts, their pathology, diagnosis and treatment.Br J Surg1924;12: 119-34.

6. Walker AR, Putnam TC. Omental, mesenteric, and retroperitoneal cysts: a clinical study of 33 new cases. Ann Surg 1973;178: 13-9.

7. Gumaste VV, Pitchumoni CS. Pancreatic pseudocyst.Gastroenterologist 1996;4: 33-43.

8. KaneMG, KrejsGJ. Pancreaticpseudocyst. Adv Intern Med 1984; 29-30.

9. Yang DM, Jung DH, Kim H, Kang JH, Kim SH, Kim $\mathrm{JH}$, et al. Retroperitoneal cystic masses: CT, clinical, and pathologic findings and literature review. Radiographics 2004; 24:1353-65.

10. Kurtz R, Heimann T, Beck R, Holt J. Mesenteric and retroperitoneal cysts. Ann Surg 1986; 203:109-12. 